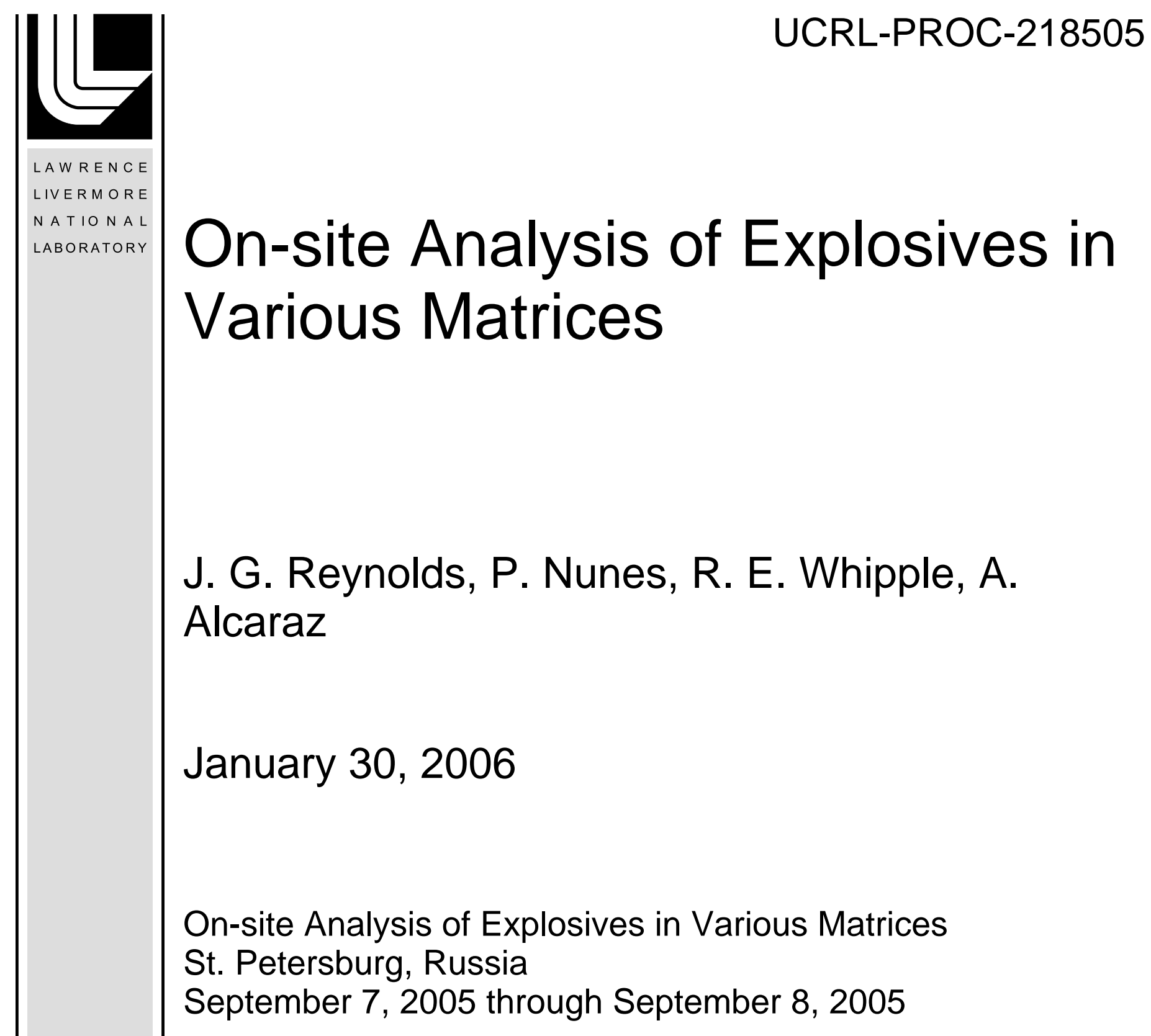


This document was prepared as an account of work sponsored by an agency of the United States Government. Neither the United States Government nor the University of California nor any of their employees, makes any warranty, express or implied, or assumes any legal liability or responsibility for the accuracy, completeness, or usefulness of any information, apparatus, product, or process disclosed, or represents that its use would not infringe privately owned rights. Reference herein to any specific commercial product, process, or service by trade name, trademark, manufacturer, or otherwise, does not necessarily constitute or imply its endorsement, recommendation, or favoring by the United States Government or the University of California. The views and opinions of authors expressed herein do not necessarily state or reflect those of the United States Government or the University of California, and shall not be used for advertising or product endorsement purposes. 


\title{
On-site Analysis of Explosives in Various Matrices
}

\author{
John Reynolds, Peter Nunes, Richard Whipple and Armando Alcaraz* \\ Lawrence Livermore National Laboratory \\ Forensic Science Center \\ 7000 East Ave, Livermore CA 94550 USA \\ alcaraz1@1ln1.gov
}

\section{Keywords:}

Trace explosives detection, on-site detection of explosives, colorimetric detection

\begin{abstract}
:
Lawrence Livermore National Laboratory (LLNL) has developed several different strategies and technologies for the on-site detection of explosives. These on-site detection techniques include a colorimetric test, thin layer chromatography (TLC) kit and portable gas chromatography mass spectrometer (GC/MS).
\end{abstract}

\section{Introduction:}

The screening of suspicious containers on-site and the search for trace explosive residue in a post-blast forensic investigation are of great importance. For these reasons, LLNL's Forensic Science Center has developed a variety of fieldable detection technologies to screen for a wide range of explosives in various matrices and scenarios. Ideally, what is needed is a fast, accurate, easy-to-use, pocket-size and inexpensive field screening test for explosives.

\section{Colorimetric Approach:}

One technology that can achieve some of these requirements is the colorimetric detection kit for explosives. As a result, LLNL has been actively developing and improving a colorimetric test for explosives, the Easy Livermore Inspection Test for Explosives (ELITE) coupon. The ELITE coupon is designed with a removable swipe to collect the questioned sample, the swipe is then placed back into the coupon holder and a Meisenheimer complex reagent (ampoule \#A) is broken. The solution then wicks across the swipe. The development of color indicates the possible presence of an explosive; if no color is produced, a Greiss Reagent (ampoule \#B) is broken. If a color develops then, it indicates the possible presence of an explosive (Figure 1).

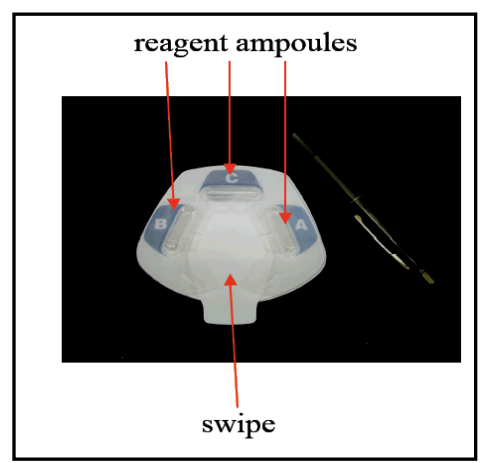

Figure 1. ELITE Coupon: Removable swipe, Meisenheimer complex regaent (ampoule \#A), Greiss Reagent (ampoule \#B) and other potential explosive indicators (ampoule \#C) 
The LLNL ELITE Coupon has been tested against a variety of common explosives at LLNL (uncertified tests), and the results are very promising (refer to Table 1).

\begin{tabular}{|c|c|c|c|}
\hline Explosive & $\begin{array}{l}\text { Color \& } \\
\text { DL (ng) }\end{array}$ & Explosive & \begin{tabular}{|l} 
Color \& \\
DL (ng)
\end{tabular} \\
\hline TNT & a 100 & 1,2-Dinitroglycerin & b 500 \\
\hline 2,4-Dinitrotoluene & a 200 & 1,3-Dinitroglycerin & b 500 \\
\hline 2,6-Dinitrotoluene & b 1000 & RDX & b 100 \\
\hline 2-Amino-4,6-dinitrotoluene & a 1000 & HMX & b 100 \\
\hline 4-Amino-2,6-dinitrotoluene & a 2000 & Dinitroethylene glycol & b 100 \\
\hline Nitroglycerin & b 100 & 1,2,4-Butanetriol trinitrate & b 100 \\
\hline 4-Nitrotoluene & b 5000 & 1,2,4-Butanetriol-1,4-dinitrate & b 100 \\
\hline Tetryl & a 100 & 1,2-Propanediol dinitrate & b 100 \\
\hline 1,3,5-Trinitrobenzene & a 50 & Nitrocellulose & b 100 \\
\hline 1,3-Dinitrobenzene & b 200 & 1-Mononitroglycerin & b 1000 \\
\hline 1,2-Dinitrobenzene & b 200 & 2-Mononitroglycerin & b 1000 \\
\hline \multirow[t]{3}{*}{ PETN } & b 100 & Sodium Nitrate & b 5000 \\
\hline & & Potassium Nitrate & b 5000 \\
\hline & & TATB & b 5000 \\
\hline
\end{tabular}

Table 1 Summary of explosives detected and their minimum quantities utilizing the ELITE coupon chemistry. The "a" on the chart represents explosives detected by the Meisenheimer complex and "b" for explosives producing a color with the Greiss Reagent.

In addition, the ELITE kit was evaluated in a field test to asses its performance under real world environmental conditions. An out of service limousine was used as the test item and several improvised devices containing varying amounts of ANFO (ammonium nitrate and fuel oil) were placed in the driver door, trunk and rear seat. Swipes taken pre and post blast (inside and outside limousine) were evaluated by ELITE. The ELITE was effective both pre-and post-blast in detecting the explosive; even the bomb technician hands tested positive after preparing and handing the improvised devices.

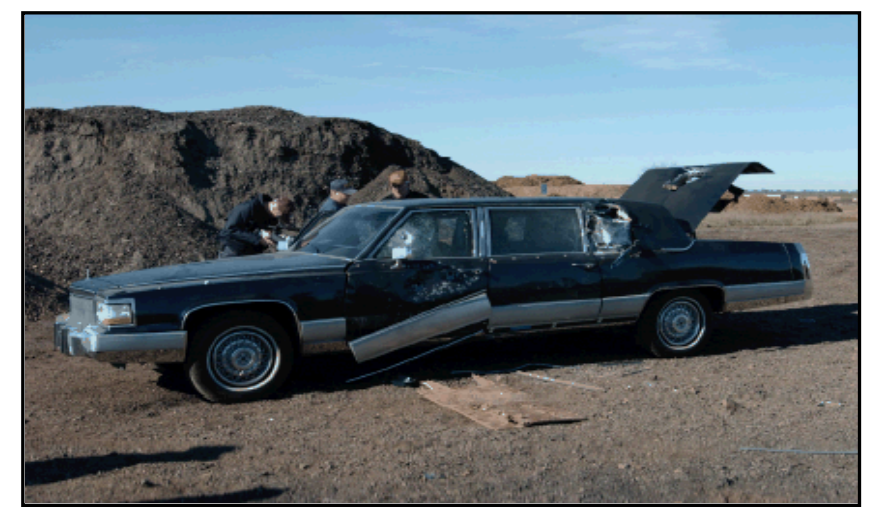

Figure 2. Out-of-service limousine utilized for testing the ELITE kit with improvised explosives devices containing ANFO (ammonium nitrate and fuel oil). 
In some situations there is a need to rapidly analyze a large number of samples in the field for the presence of explosives. For this scenario, TLC is an ideal technology. It is a sensitive technique that makes use of the migration of compounds within a solvent and coated TLC plate to obtain the separation of complex mixtures (both unknowns and explosives reference standards) for explosives identification. After the compounds have migrated on the TLC plate, color reagents are sprayed onto the plates to highlight target compounds, Figure 3. With a basis of classic TLC, LLNL has modernized the technique by incorporating digital technology into the kit. For example, a digital camera/light box takes an image of the processed TLC plate, the image is transferred to a lap top computer and a software program compares the migrations time of the reference materials (explosives standards) and the associated colors with the analyte "spots'. This LLNL TLC kit has been evaluated in field deployments (Figure 4).

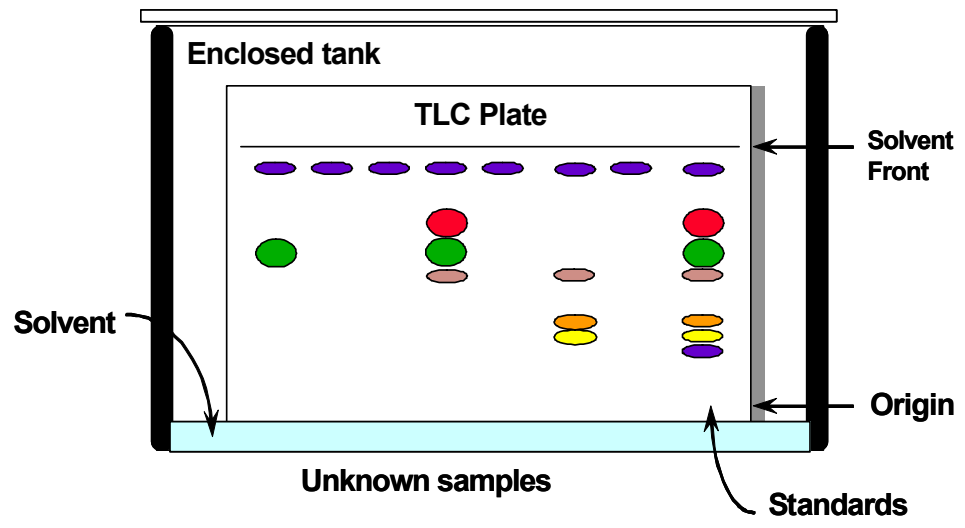

Figure 3. Protocol that makes use of the migration of compounds with a solvent and coated TLC plate to obtain the separation of complex mixtures.
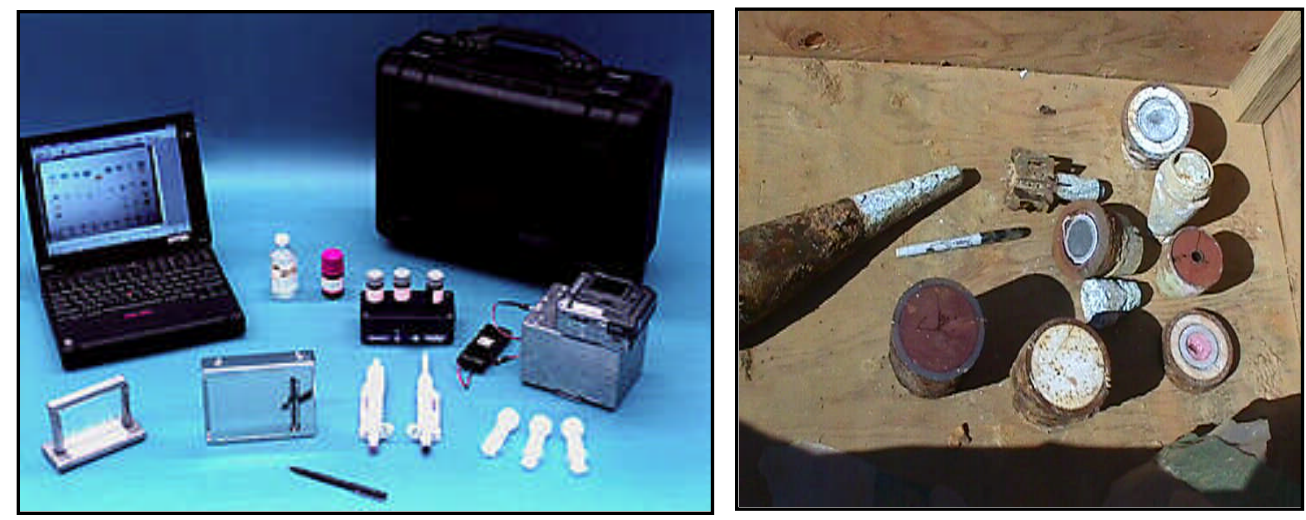

Figures 4. LLNL TLC kit (right) and unearthed munitions screened for explosives utilizing the LLNL TLC kit. 
Portable Gas Chromatography/Mass Spectrometer (GC/MS) Approach:

One technology that can achieve greater specificity and detect a wider range of threat materials is a portable GC/MS system. LLNL and other research groups have been developing various portable GC/MS instruments for several years. LLNL, however, has developed a fully functional, laboratory-performance-based system that can be deployed to field locations for analysis of complex organic samples. The LLNL system incorporated an Agilent quadrupole mass analyzer, light weight vacuum chamber, on-board carrier gas system, and unique gas chromatography oven (25-310 C), Figure 5. The portable GC/MS has been deployed to various areas for evaluation and in one instance it was deployed to a bomb evidence training site to collect and analyze swipes pre-and post-loading of explosives into the vehicle. The portable GC/MS was able to successfully identify PETN (detonation cord utilized for the ANFO device) in the post loaded vehicle, Figure 6.

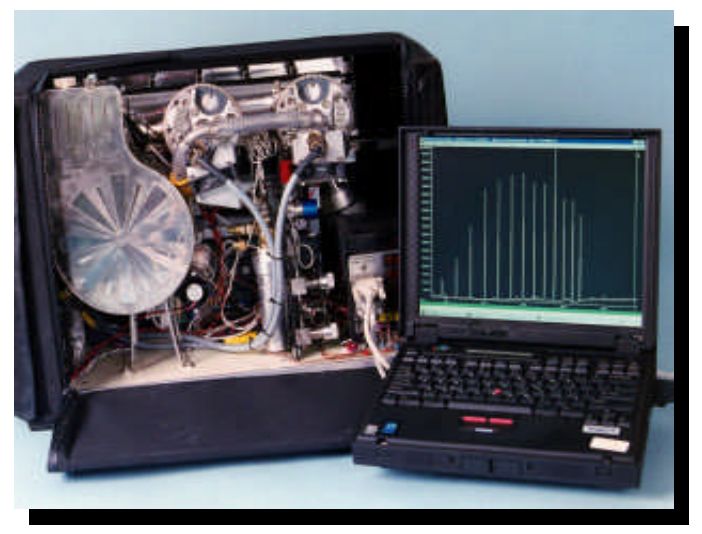

Figures 5. LLNL portable GC/MS system; weight approximately 70 pounds

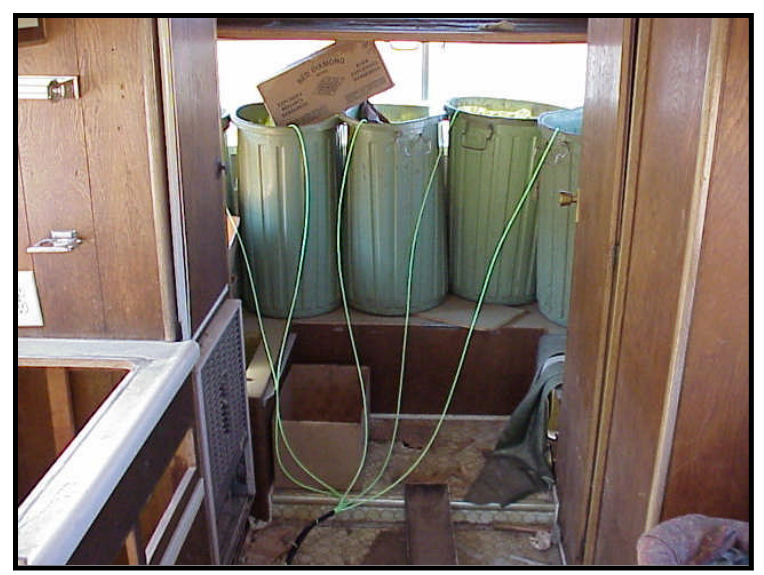

Figures 6. Vehicle loaded with explosives (ANFO) and PETN detonation cord; swipes analyzed by LLNL portable GC/MS system confirmed the presence of PETN and fuel oil. 


\section{Summary of Detection Approaches:}

The selection of a particular on-site analytical detection technique for explosives must be systematically evaluated for possible unforeseen user scenarios. For example, the effects of humidity, heat, and cold or high levels of environmental chemical background may impede the detection technology or even render it ineffective. For this reason, it is advantageous to utilize serval detection systems. Below are summarized advantages of utilizing a suite of explosive detection technologies developed by the LLNL FSC:

\section{Colorimetric Test for Explosives (ELITE)}

- Rapid screen for explosives in the field

- Requires less than 2 minutes per analysis

- Kit weight less than $5 \mathrm{lbs}$.

- Nano-gram detection limit for most explosives

- To date: Detects 26 explosives

- New ELITE coupon, less expensive and lightweight

\section{Thin Layer Chromatography (TLC)}

- Rapid analysis of a large number of samples

- Easy to use, low cost

- Requires 25 minutes for 10 samples

- New reagents needed, smaller kits

- Nano-gram detection for most explosives

- To date: detects 25 explosives

\section{Portable GC/MS}

- Identification of questioned samples in the field

- 25 minutes per analysis

- Requires extensive training

- Higher maintenance costs

- External power (generator)

\section{References:}

1) Lawrence Livermore National Laboratory, Science and Technology Review, Forensic Science Center, April 2002 page 11-18.

This work was preformed under the auspices of the U.S. Department of Energy by UC, Lawrence Livermore National Laboratory under contract W-7405-ENG-48.

$$
-0-0-0-0-0-
$$

